8 Buckingham B A, Uitto J, Sandborg C, Keens T, Kaufman F, Landing B. Scleroderma-like syndrome and the non-enzymatic glucosylation of collagen in children with poorly controlled insulin-dependent diabetes (IDDM). Pediatr Res 1981; 15: 626.
Correspondence to Dr J E H Brice, Green Wood Children's Centre, City Hospital Hucknall Road, Nottingham NG5 1PD.

Received 16 July 1982

\title{
Congenital generalised fibromatosis
}

\author{
NEENA MODI \\ Medical Paediatric Unit, Western General Hospital, Edinburgh
}

SUMMARY A case of congenital generalised fibromatosis is described. The need to avoid unnecessary treatments is stressed.

Congenital generalised fibromatosis is a rare disease which presents in the newborn period. Multiple nodules of fibrous tissue are formed in muscle, bone, subcutaneous tissue, and viscera. Only 33 cases have been described.

\section{Case report}

A baby girl, delivered at term, was found on routine initial examination to have a rubbery $3-\mathrm{cm}$ nodule overlying the posterior iliac crest. In addition, five $3-\mathrm{cm}$ circular, pigmented macules were scattered over the back and buttocks. There was no lymphadenopathy or hepatosplenomegaly and the baby was clinically well. Her 25-year-old mother had been in good health throughout pregnancy and exposed to no drugs or toxic substances. On the baby's second day of life another nodule appeared and by the next day there were 2 more. The pigmented macules darkened and new lesions appeared. The original nodule increased in size to $9 \mathrm{~cm}$ in diameter. All investigations including biochemistry, haematology, bone marrow, and radiographs of the chest were normal. The largest tumour was excised but apart from confirming that it was locally invasive the histopathology proved difficult to interpret. For this reason tumour sections were sent to several centres around the world. The sections showed pronounced variation from field to field. The non-encapsulated, cellular neoplasm was predominantly fibroblastic but contained numerous thin-walled vascular spaces. Areas of necrosis were present. Some areas had a predominantly angiomatous appearance and some a neural appearance.

In view of the uncertainty about their nature, and the fact that the tumours were known to be locally invasive, the decision whether to recommend multiple excision remained. However on the baby's seventh day one of the nodules appeared to be decreasing in size; by the end of the second week there was no doubt about the clinical regression. At age one month the baby developed a nappy rash with superimposed staphylococcal sepsis; the nodules continued to regress. At 2 months no palpable nodules remained. At 3 months she required admission for severe seborrhoeic dermatitis and diarrhoea, but responded well to conservative treatment. At 4 months her mother attempted suicide in the belief that her child was suffering from cancer. At 7 months she remains well, with no evidence of active disease. Biochemistry, haematology, serum immunoglobulins, bone scan, and skeletal survey are normal.

The pathological opinions received included the following diagnoses: 'fibrous type tumour', haemangioendothelioma, haemangiopericytoma, angiomatous mesenchymoma, and congenital generalised fibromatosis. A search of the literature confirmed that both on clinical and histopathological grounds a diagnosis of congenital generalised fibromatosis could be made.

\section{Discussion}

Congenital generalised fibromatosis, although rare, is well defined clinically and histologically. Of the 33 cases cited, the majority come from America and a few from France, Germany, and Australia. ${ }^{1-3}$ The term was first used by Stout in 1954 to describe two rapidly fatal cases. Eleven years later Kauffman and Stout, reviewing their own cases, distinguished two groups dependent on the presence or absence of visceral involvement. ${ }^{4}$ In both groups presentation is in the newborn period with widespread, multiple, rubbery nodules. There is often a dominant nodule; solitary lesions also occur. ${ }^{5}$ The clinical course of the lesions is to enlarge initially but ultimately to regress. In the so-called generalised group (with visceral involvement) survival is poor and death, invariably in the first year of life, owing to the involvement of vital structures during the phase of active growth and regression, producing for example, respiratory failure, ${ }^{3}$ intestinal obstruction, ${ }^{2}$ renal masses, ${ }^{6}$ and 
myocardial lesions. ${ }^{7}$ No nodules in the central nervous system have been described. Cases without visceral nodules (the multiple group) demonstrate a wide range of involvement from single bony or subcutaneous nodules to widespread involvement of skin, subcutaneous tissue, muscle, and bone. As ultimate regression is the rule survival is assured.

The difficulty in making a pathological diagnosis lies in the variability of appearances from field to field on microscopical examination. ${ }^{7}$ Multiple foci of fibroblastic tissue occur; these are locally invasive and not encapsulated. Mitotic figures are sometimes present and this gives rise to the most common misdiagnosis of fibrosarcoma. However the cells are well differentiated. In keeping with the tendency to spontaneous regression, areas of degenerative change-such as hyalinisation, calcification, and necrosis-are always present. Vascular channels are prominent, but there may be a variety of tissue elements, raising the possibility of hamartomatous rather than neoplastic formation. The bony lesions may cause diagnostic confusion, especially if they are the presenting feature. They are lytic, well marginated, show varying degrees of sclerosis, ${ }^{16}$ and may result in pathological fractures. The nodules are thought to represent multiple foci and not metastases from a single source. ${ }^{7}$

Although the aetiology of congenital generalised fibromatosis is unknown, the condition may be an abnormal response to oestrogen stimulation. The lesions develop in utero and ultimately regress when removed from the uterine environment. Early cancer researchers found that guinea-pigs developed multiple fibrous tumours if exposed to prolonged oestrogen stimulation and that when treatment was stopped the tumours regressed. Against this is the fact that the pigmented 'haemangiomas' or macules noted in this case and that of others ${ }^{3}{ }^{8}$ may represent the remains of nodules that have actually regressed in utero.

Although the cause is unknown the diagnosis may be confidently made on clinical and pathological features. Prognosis rests on a careful search for visceral involvement. Survival with visceral involvement is possible but unlikely. As fibrous tumours respond poorly to chemotherapy, the value of this approach is doubtful. In the absence of visceral involvement the outlook is excellent and mutilating and harmful treatment must be avoided.

I thank Mrs Catriona Lawrie for secretarial assistance, Dr A D Bain for pathology, and Dr A J Keay for permission to report this case.

\section{References \\ 1 Baer J W, Radkowski M A. Congenital multiple fibro- matosis. A case report with review of the world literature. AJR 1973; 118: 200-5. \\ ${ }^{2}$ Lin J J, Svoboda D J. Multiple congenital mesenchymal tumors. Multiple vascular leiomyomas in several organs of a newborn. Cancer 1971; 28: 1046-53. \\ 3 Roggli V L, Han-Seob Kim, Hawkins E. Congenital generalized fibromatosis with visceral involvement. A case report. Cancer 1980; 45: 954-60. \\ 4 Kauffman S L, Stout A P. Congenital mesenchymal tumors. Cancer 1965; 18: 460-76. \\ 5 Briselli M F, Soule E H, Gilchrist G S. Congenital fibro- matosis. Report of 18 cases of solitary and 4 cases of multiple tumors. Mayo Clin Proc 1980; 55: 554-62. \\ 6 Teng P, Warden M J, Cohn W L. Congenital generalized fibromatosis (renal and skeletal) with complete spon- taneous regression. J Pediatr 1963; 62: 748-53. \\ 7 Shnitka T K, Asp D M, Horner R H. Congenital general- ized fibromatosis. Cancer 1958; 11: 627-39. \\ 8 Beatty E C. Congenital generalized fibromatosis in infancy. Am J Dis Child 1962; 103: 620-4.}

Correspondence to Dr N Modi, Department of Paediatrics, Neonatal Unit, University College Hospital, Gower Street, London WC1E 6AU.

Received 30 June 1982

\section{Infant mortality in developing countries}

\section{A ASHWORTH AND J C WATERLOW}

\section{Department of Human Nutrition, London School of Hygiene and Tropical Medicine}

SUMMARY In developing countries, mortality among the under-fives is greatest during infancy. Limited data indicate that about $80 \%$ of these infant deaths occur in the first 6 months of life and two-thirds of the postneonatal deaths occur before 6 months. These findings strongly suggest that more attention should be focused on the causes of death in the very young infant.
In the last few years it has become increasingly clear how wrong it is to group together infants and preschool children as if they formed a homogeneous 'vulnerable' group from 0-5 years. This is particularly true with regard to malnutrition. Longitudinal studies in the Punjab have shown that for a given nutritional status, the younger the child the greater is the risk of death. ${ }^{1}$ The pattern of malnutrition also changes during the first 5 years of life. ${ }^{2}$ Wasting (that 